Proc. Indian Acad. Sci. (Chem. Sci.), Vol. 105, No. 6, December 1993, pp. 475-486.

(C) Printed in India.

\title{
Cis-trans photoisomerization of 1,2-diarylethylenes: Effect of charge transfer interactions
}

\author{
U MAZZUCATO*, G G ALOISI and F ELISEI \\ Dipartimento di Chimica, Università di Perugia, I-06123 Perugia, Italy
}

\begin{abstract}
This paper describes the results of an extensive study of trans $\rightarrow$ cis photoisomerization of various 1,2-diarylethylenes (DAE). The structure effect on the competitive radiative and reactive deactivations and on the isomerization mechanism (singlet/triplet, diabatic/adiabatic) is discussed. The effect of charge-transfer (CT) interactions of DAE with electron donors and acceptors are then presented. Triplet-induced isomerization is generally the common effect in non-polar solvents, and is particularly efficient when a heavy atom is involved. Typical examples of CT-induced triplet photoisomerization of DAE by electron donors and acceptors are llustrated. New results on some fluorescent cis compounds are also reported. Prevalent radical ion formation in polar solvents is shown to generally quench isomerization from trans to $c$ is but to induce isomerization from cis to the more stable trans radical ion.
\end{abstract}

Keywords. Photoisomerization; diarylethylenes; charge-transfer; triplet induction; radical ions.

\section{Introduction}

Stilbene analogues, the 1,2-diarylethylenes (DAE), have been the object of deep investigations during the last decade. Sustained interest in such molecules lies in the fact that they are simple models for the study of cis-trans photoisomerization around the ethylenic bond (Saltiel and Charlton 1980; Saltiel and Sun 1990) and of s-cis-s-trans (photo)isomerization around the quasi-single bond between the aryl and the ethylenic bridge (Mazzucato and Momicchioli 1991) as well as in the inherent importance of these processes for applicative research (photography, photochromism, processes of biological interest such as vision etc.).

Recent studies on photoisomerism of DAE have shown that the excited-state properties depend to a large extent on the size and nature of the aryl groups linked to the ethylenic bridge (Bartocci et al 1992; Mazzucato 1982, 1987).

Greater attention has been paid to the trans isomers, where fluorescence, isomerization to cis in $S_{1}$ (singlet mechanism) and intersystem crossing (ISC) (eventually followed by isomerization in $T_{1}$, triplet mechanism) are the competitive deactivation channels of the $S_{1}$ state. In the parent molecule, stilbene (S), the avoided crossing between the $S_{1}\left(B_{u}\right)$ energy curve as a function of the twist angle around the central double bond (with the energy increasing by twisting towards $90^{\circ}$, at the perp

* For correspondence 
configuration) and the upper (doubly excited?) $S_{n}\left(A_{g}\right)$ curve (with an "ethylenic" minimum in the perp configuration) leads to the well known and widely accepted diabatic isomerization mechanism in $S_{1}$. From the trans side, this pathway involves an activated ${ }^{1}$ trans ${ }^{*} \rightarrow{ }^{1}$ perp ${ }^{*}$ twisting towards the energy minimum, followed by internal conversion to the ground state configuration, ${ }^{G}$ perp, and almost 50:50 partitioning to the stable trans and cis isomers (Saltiel and Charlton 1980; Saltiel and Sun 1990). When a phenyl group (P) of stilbene is replaced by larger polycyclic aryl groups of gradually decreasing $S_{1}$ and $T_{1}$ energy, such as naphthyl $(\mathrm{N})$, phenanthryl (Ph), chrysenyl (C), pyrenyl (Py) and anthryl (An) groups, the $S_{1}$ curve shifts towards lower energies (more in the trans and cis than in the perp geometry) while the $S_{n}$ curve is less affected by the aryl substitution. Therefore, the $S_{1}-S_{n}$ crossing is no longer possible and a maximum, instead of a minimum, is present at $90^{\circ}$ in the $S_{1}$ energy curve. The activation energy to twisting in $S_{1}$ does gradually increase (Mazzucato 1982, 1987), thus leading to a decrease in the isomerization quantum yield in $S_{1},{ }^{1} \phi_{t \rightarrow c}$, and can become insuperable at room temperature. The competitive processes of fluorescence (possibly accompanied by some internal conversion) and ISC are then much more favoured, their relative amounts being determined by the nature of the aryl groups (Bartocci et al 1992). When ISC is substantial, the triplet mechanism can maintain an overall high isomerization yield, unless high barriers to ${ }^{3}$ trans ${ }^{*} \rightarrow{ }^{3}$ perp ${ }^{*}$ twisting completely inhibit the trans to cis isomerization, as in the case of the anthryl derivatives ("one-way" isomerization, only from cis to trans) (Sandros and Becker 1987; Arai et al 1988; Tokumaru and Arai 1992). A suitable choice of the aryl groups linked to the central ethylenic bridge allows the gradual tuning of the cis-trans photoisomerization from a singlet to a triplet pathway and finally the inhibition of the reaction in the trans $\rightarrow$ cis way.

From the cis side, the torsional barriers are always lower compared with those from the trans side. Therefore, when a maximum instead of a funnel is present at the ${ }^{1}$ perp* configuration, a direct adiabatic mechanism $\left({ }^{1}\right.$ cis $^{*} \rightarrow{ }^{1}$ trans $\left.{ }^{*}\right)$ can become operative in both the singlet (Spalletti et al 1991; Mazzucato et al 1993) and triplet (Arai et al 1988; Tokumaru and Arai 1992) manifolds.

The fluorescence of trans-DAE is quenched by charge transfer (CT) interactions with electron donors (e.g., amines) or acceptors (e.g., cyano-aromatics) and exciplex formation, as evidenced by their typical emission bands, is observed in non-polar solvents (Aloisi et al 1980, 1988; Aloisi and Elisei 1990; Elisei et al 1992). Singletinduced CT interactions may result in enhanced formation of DAE triplets in non-polar solvents (Elisei et al 1990; Aloisi et al 1992; Görner et al 1992) and DAE radical ions in polar solvents (Aloisi et al 1991, 1992; Görner et al 1992). The extent of the quenching, which depends on the nature of the two partners and the solvent, may then affect both the yield and the mechanism of photoisomerization (Aloisi et al 1980; Aloisi and Elisei 1990; Aloisi et al 1988, 1991; Elisei et al 1992).

On the basis of published data obtained from the spectroscopic and photochemical study of a large series of DAE as well as of some new results recently obtained in our laboratory, the main features of the structure effect on photoisomerism of DAE are reported here. The CT interactions of the fluorescent trans isomers and some cis analogues with electron donors and acceptors will then be discussed to evidence their effects on the excited state behaviour of DAE. 


\section{Experimental}

All compounds were synthesized by standard procedures as per previous work. Fluorimetric, photochemical and flash photolytic measurements were made as described in previous papers (see Bartocci et al 1987 and Aloisi et al 1992, and papers cited therein). Benzene, $n$-hexane, methylcyclohexane (MCH), chlorobenzene (CB), ethylacetate (EtAc) and acetonitrile (MeCN) from Carlo Erba (RPE grade) were used as solvents, tributhylamine (TBA), diethylaniline (DEA), 4-bromo-N, N-dimethylaniline (BrDMA) from Fluka $A G$ and potassium iodide from Carlo Erba as electron donors, and chloranil (Chl) from Fluka $A G$ and dicyanobenzene (DCNB) from EGA-Chemie as electron acceptors.

\section{Results and discussion}

\subsection{Photoisomerization}

Figure 1 shows a qualitative sketch (a), based on theoretical and experimental data, of the torsional potential energy of the $S_{1}$ state of three typical DAE together with a scheme (b) of the energy levels of the lowest singlet excited state of the DAE and of their arene $\left({ }^{1} L_{a},{ }^{1} L_{b}\right)$ and ethylene $\left({ }^{1} B_{u}\right)$ chromophores (Bartocci et al 1992). Perturbation coupling of the excited state localized on the largest arene chromophore

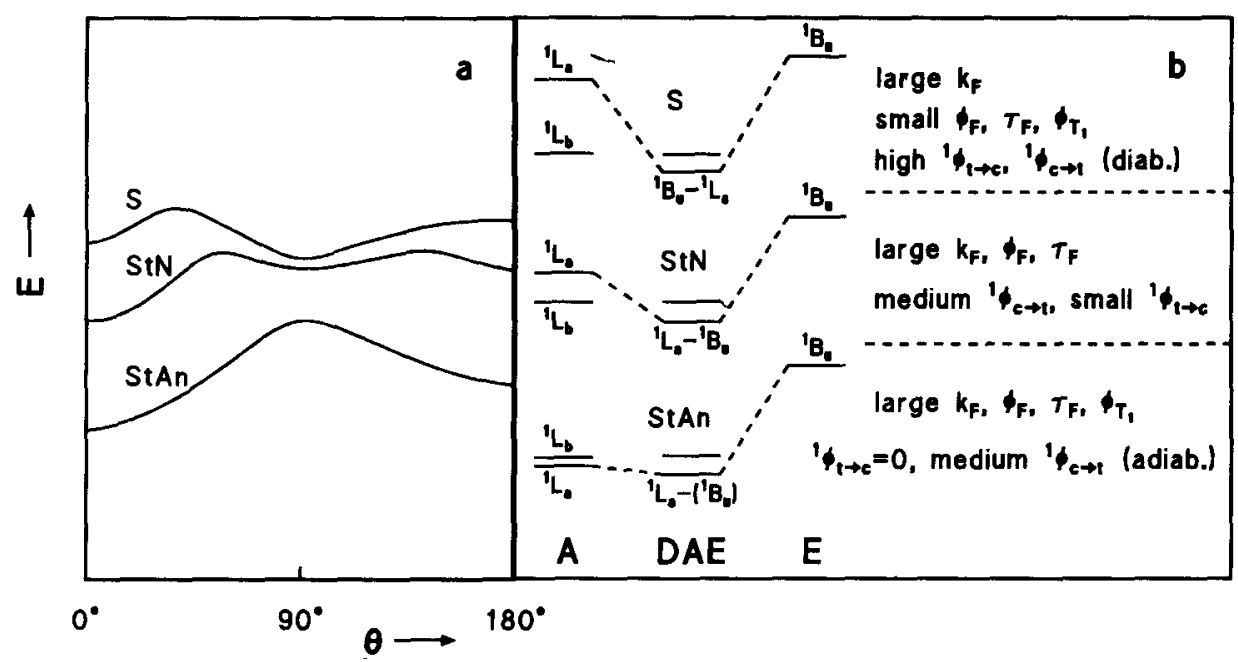

Figure 1. (a) Sketch of the torsional energy barrier for the $S_{1}$ state of stilbene (S: singlet mechanism), styrylnaphthalenes (StN: prevalent singlet mechanism at room temperature, mixed singlet + triplet mechanism at lower temperatures) and styrylanthracenes (StAn: no trans $\rightarrow$ cis isomerization); (b) sketch of the interactions between the lowest excited states $L_{a}$ and $L_{b}$ of the polycyclic aryl chromophore (A) and the $B_{u}$ state of ethylene (E) to give the resulting $S_{1}$ state of the trans-DAE (the effect of the relative positions of the energy levels on the fluorescence rate parameter, quantum yield and lifetime and on the photoisomerization quantum yields are also indicated). 
(particularly of ${ }^{1} L_{a}$, which has a higher transition moment) with the ${ }^{1} B_{u}$ state of ethylene gives rise to lowest excited states of different character for the DAE molecules.

When the energy of the arene ${ }^{1} L_{a}$ state is high, not much lower than that of the lowest excited state ${ }^{1} B_{u}$ of ethylenic character, the interaction is strong leading to a mixing of local states with a large contribution of the ethylenic $\pi, \pi^{*}$ state to the $S_{1}$ state of the DAE. A high ${ }^{1} \phi_{t \rightarrow c}$ and low $\phi_{F}$ is expected in this case, as found for trans-S.

When the energy of ${ }^{1} L_{a}$ decreases markedly (naphthyl, phenanthryl derivatives), the DAE excitation energy is largely localized in the aryl (prevalent $L_{a}$ character) and a high torsional barrier in $S_{1}$ leads to a small ${ }^{1} \phi_{t \rightarrow c}$. However, in some cases, particularly when the lowest excited state is the arylic ${ }^{1} L_{b}$ state (weak $S_{0} \rightarrow S_{1}$ transition and low extinction coefficients), ISC can become substantial opening the triplet pathway to isomerization.

A limiting situation is reached when ${ }^{1} L_{a}$ (lying in such cases below ${ }^{1} L_{b}$ ) is so far apart from ${ }^{1} B_{u}$ that the mixing is very weak $\left(S_{1}\right.$ of aromatic character, as in anthryl derivatives). The barriers to twisting are high in both $S_{1}$ and $T_{1}$ and no isomerization occurs from trans to cis but only from cis to trans ("one-way" isomerization, Arai et al 1988, Tokumaru and Arai 1992). The presence of a maximum, instead of a minimum, at the ${ }^{1}$ perp ${ }^{*}$ configuration for the pyrene and anthracene derivatives causes the activated twisting of ${ }^{1}$ cis $^{*}$ to directly produce ${ }^{1}$ trans*, thus favouring an adiabatic photoisomerization mechanism, as reported for the triplet state (Arai et al 1988; Tokumaru and Arai 1992), particularly in polar solvents where the activation energy in $S_{1}$ is lowered because of the stabilization of the zwitterionic twisted configuration (Spalletti et al 1991; Mazzucato et al 1993).

Figure 2 shows the increase in the fluorescence quantum yield $\left(\phi_{F}\right)$ and the decrease in the trans $\rightarrow$ cis photoisomerization quantum yield $\left(\phi_{t \rightarrow c}\right)$ of some styryl-arenes on increasing the size of the arene and the aromatic character of the $S_{1}$ state of the DAE. Table 1 shows how the nature of the largest arene affects the photoisomerization mechanism (singlet/triplet, diabatic/adiabatic). Stilbene isomerizes with high yield via a singlet diabatic mechanism (Saltiel and Charlton 1980; Saltiel and Sun 1990). The

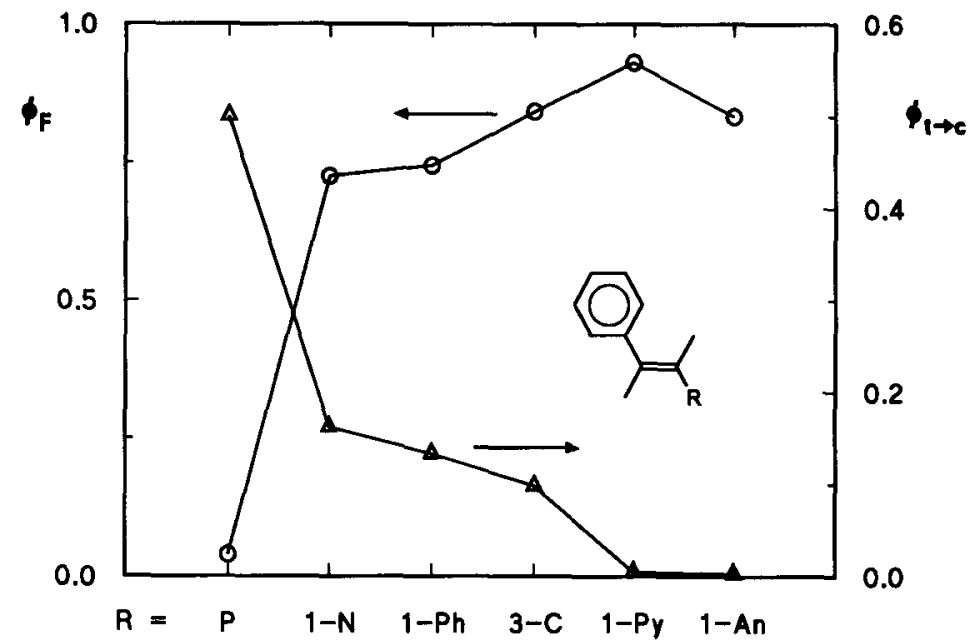

Figure 2. Effect of the nature and size of the aryl substituent of styrene on the fluorescence $(O)$ and trans $\rightarrow$ cis photoisomerization $(\Delta)$ quantum yields of some DAE. 
Table 1. Fluorescence, triplet and isomerization quantum yields and prevalent isomerization mechanism for some typical trans-DAE in non-polar solvents at room temperature.

\begin{tabular}{|c|c|c|c|c|}
\hline Compound & $\phi_{F}$ & $\phi_{T}$ & $\phi_{t \rightarrow c}$ & Prevalent mechanism \\
\hline $\mathbf{S}^{\mathbf{a}}$ & 0.04 & $<0.01$ & 0.50 & Singlet, diabatic \\
\hline $1-S_{t} N^{b}$ & 0.72 & 0.04 & 0.16 & Singlet, diabatic \\
\hline 1-StPhe & 0.61 & 0.18 & $0 \cdot 14$ & Triplet, diabatic \\
\hline $2-\mathrm{StPh}^{\mathrm{c}}$ & 0.35 & $<001$ & 0.24 & Upper triplet, diabatic \\
\hline 9-StPh ${ }^{\mathrm{c}}$ & 0.67 & 0.32 & 0.13 & Mixed, diabatic \\
\hline 1-StPy & 0.94 & $<0.03$ & $<0.02$ & Only cis $\rightarrow$ trans: singlet, adiabatic \\
\hline 9-StAnd & 0.44 & 0.32 & - & Only cis $\rightarrow$ trans: mixed, adiabatic \\
\hline
\end{tabular}

References: ' Saltiel and Charlton (1980); ${ }^{b}$ Elisei et al (1989), ' Aloisi and Elisei (1990); Elisei et al (1990); ' Bartocci et al (1992).

$n$-styrylnaphthalenes (n-StNs) (Bartocci et al 1984; Elisei et al 1989) have a prevalent radiative deactivation of $S_{1}$ : they isomerize by the same mechanism as $S$ above room temperature but follow a prevalent triplet pathway below $270 \mathrm{~K}$ (or at slightly lower temperatures in polar solvents). The $n$-styrylphenanthrenes ( $n$-StPhs) (Bartocci et $d$ 1987) follow a mixed singlet/triplet mechanism already at room temperature due to higher torsional barriers in $S_{1}$ and larger triplet yields. In some cases (e.g., in 2-StPh), upper $S_{n}$ or $T_{n}$ states of ethylenic character may play a role in the isomerization favouring a fast twisting which by-passes the lowest excited states (Elisei et al 1990, Aloisi et al 1991a). In fact, 2-StPh has a high torsional barrier in $S_{1}$ and a $\phi_{t \rightarrow c}$ value as large as 0.24 which would indicate that at least $\sim 50 \%$ of the excited molecules deactivate through a trans $\rightarrow$ perp twisting in the triplet manifold. However, $T_{1}$ cannot be an intermediate since its yield is smaller than 0.01 . Therefore, one could think of an isomerizable upper $T_{n}$ state, almost isoenergetic with $S_{1}$. In fact, at low temperatures, when twisting in $T_{n}$ is slowed down by the viscosity barrier, the population of $T_{1}$ increases substantially by $T_{n} \rightarrow T_{1}$ internal conversion and the lowest excited triplet state becomes readily detectable. A similar mechanism was hypothesized for the isomerization of 4-halogenated stilbenes (Görner and Schulte-Frohlinde 1979). In any case, the presence of a shallow minimum in the ${ }^{1}$ perp* configuration of StPhs still seems to favour the diabatic mechanism. Practically, the 1-styrylpyrene (StPy) and $n$-styrylanthracenes (StAns) do not isomerize from trans to cis because of very high torsional barriers in both $S_{1}$ and $T_{1}$. They follow a mixed (singlet/triplet) mechanism from cis to trans with a prevalent adiabatic character.

In conclusion, the singlet mechanism is favoured at high temperatures and in polar solvents; the adiabatic mechanism is favoured in the presence of a maximum at the ${ }^{1,3}$ perp* configurations, particularly in polar solvents where the activation energy is smaller.

\subsection{Effect of CT interactions on trans $\rightarrow$ cis photoisomerization}

The trans $\rightarrow$ cis isomerization quantum yield $\left(\phi_{t \rightarrow c}\right)$ in the presence of the fluorescence quencher $Q$ is the sum of two contributions, by the monomer (non-complexed) molecules $\left(\phi_{\mathrm{CM}}\right)$ and by those which react through the exciplex $\left(\phi_{\mathrm{CE}}\right)$. When $[Q] \rightarrow \infty$, 
the fluorescence and photoisomerization quantum yields of the exciplex reach their limiting values, $\phi_{\mathrm{FE}}^{\mathrm{lim}}$ and $\phi_{\mathrm{CE}}^{\mathrm{lim}}$, respectively.

Often, the overall photoisomerization is less quenched than fluorescence or even enhanced by addition of electron donors or acceptors since CT-induced ISC can lead to isomerization in the triplet manifold. In fact, since both $S_{1}$ and $T_{1}$ are potentially reactive states (the actual reactivity depending on their torsional barriers), the expected effect is generally modest. Since the quenching efficiency of the singlet-excited transDAE by electron donors or acceptors depends on the redox potential of the two partners, a suitable choice of the additive is required for an efficient CT-induced photoisomerization, based on the following criteria: high quenching rate constant $k_{\mathrm{Q}}$, low yields of both the radiative decay $\left(\phi_{\mathrm{FE}}\right)$ and the internal conversion to the ground state $\left(\phi_{\mathrm{GE}}\right)$ and high ISC yield $\left(\phi_{\mathrm{TE}}\right)$ of the exciplex so as to induce a substantial population of the reactive triplet state of the olefin.

Figure 3 shows, as an example, the decreasing of fluorescence intensity of 9-StPh together with the parallel increase of the triplet and isomerization yields in the case of the interaction with DEA as donor in $n$-hexane. Table 2 shows some typical cases

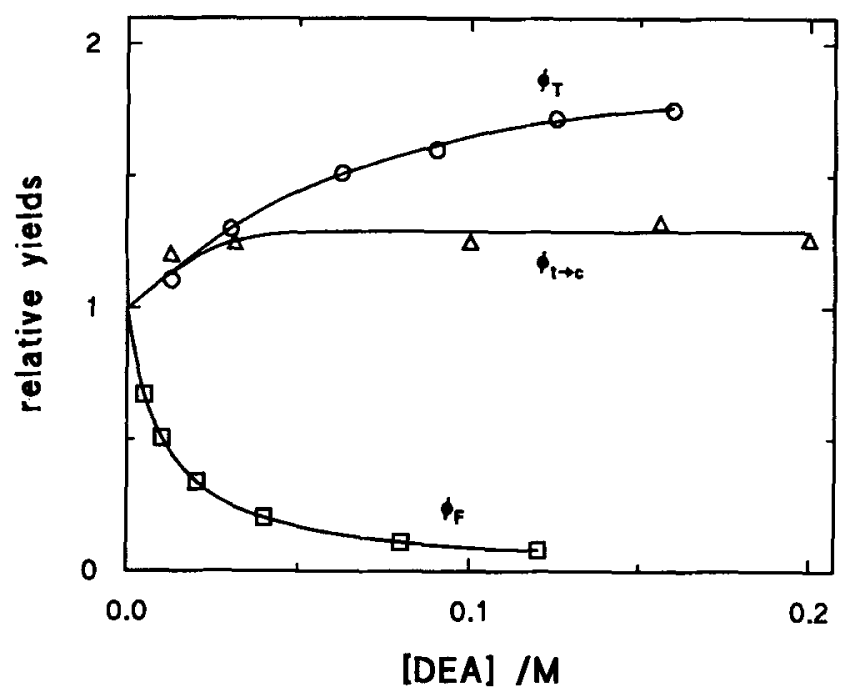

Figure 3. Effect of DEA concentration on the quantum yields of fluorescence (squares), photoisomerization (triangles) and triplet (circles) for trans-9-StPh/DEA in $n$-hexane.

Table 2. Limiting ([Q] $\rightarrow \infty$ ) fluorescence and trans $\rightarrow$ cis photoisomerization quantum yields of some typical DAE in the presence of three amines as donors in $n$-hexane and DCNB as acceptor in benzene.

\begin{tabular}{|c|c|c|c|c|c|c|c|c|c|c|}
\hline \multirow[b]{2}{*}{ DAE } & \multirow[b]{2}{*}{$\phi_{F}$} & \multirow[b]{2}{*}{$\phi_{t \rightarrow c}$} & \multicolumn{4}{|c|}{$\phi_{\mathrm{FE}}^{\mathrm{lim}}$} & \multicolumn{4}{|c|}{$\phi_{\mathrm{CE}}^{\lim }$} \\
\hline & & & TBA & DEA & BrDMA & DCNB & TBA & DEA & BrDMA & DCNB \\
\hline 2-StN & $0.65^{a}$ & $0 \cdot 12^{\mathrm{a}}$ & $0 \cdot 17^{a}$ & $0.72^{\mathrm{b}}$ & & $0.17^{\mathrm{c}}$ & $0 \cdot 10^{a}$ & $0 \cdot 12^{b}$ & & $0.26^{c}$ \\
\hline $1-\mathrm{StPh}^{\mathrm{d}}$ & 0.61 & 0.14 & 0.19 & 0.36 & $\sim 0.01$ & & $0 \cdot 10$ & 0.20 & 0.44 & \\
\hline 9-StPh & $0.67^{d}$ & $0.13^{d}$ & $0 \cdot 17^{\mathrm{d}}$ & $0.57^{d}$ & $<0.01^{d}$ & $0.37^{\mathrm{c}}$ & $0.11^{d}$ & $0 \cdot 10^{d}$ & $0.28^{d}$ & $0 \cdot 24^{c}$ \\
\hline $2,3^{\prime}$-NPE & $0.67^{a}$ & $0 \cdot 15^{\mathrm{a}}$ & $0.03^{a}$ & & & $0.24^{\mathrm{c}}$ & $0.09^{\mathrm{a}}$ & & & $0.34^{c}$ \\
\hline
\end{tabular}


of the limiting $([Q] \rightarrow \infty)$ quantum yields of radiative and reactive deactivation of the exciplexes with three amine donors and one acceptor in non-polar solvents. Chromatographic control showed that the formation of photoproducts different from the cis isomers, as observed for the S/amine systems (Lewis 1979; Lewis and Simpson 1979; Lewis et al 1992), is generally negligible in our experimental conditions. Perusal of table 2 shows that substantial isomerization is induced by DCNB in benzene where higher triplet yields were measured even if accompanied by substantial yields of radiative decay of the exciplex. On the other hand, radiative decay is the prevalent deactivation pathway of the exciplex with DEA and internal conversion is the prevalent one with the less efficient TBA quencher. For the exciplex of 9-StPh, quenching rate constants, $k_{\mathrm{O}}$, of 9.2 and $3.4\left(10^{9} \mathrm{M}^{-1} \mathrm{~s}^{-1}\right)$ were obtained with DEA and TBA, respectively. As expected, larger induction was obtained with BrDMA. Inclusion of a heavy atom in the solvent or, even better, in one of the two partners increases the isomerization yield because the spin-orbit coupling controlled by the exciplex produces the isomerizable $T_{1}$ state. In this case $\phi_{\mathrm{FE}}$ is very low (smaller than 0.01 ) but enough to demonstrate the existence of the complex and then of a CT-assisted spin-orbit coupling effect.

The data above allow some conclusions to be drawn about the CT effect on the photoisomerization of trans-DAE in non-polar solvents. When both the lowest excited states of the olefin can be effectually reactive (as in S and many other DAE) or nonreactive (as in n-StAns), the effect of the CT interaction is expected to be zero or very small (both positive and negative), the quenching of $S_{1}$ being generally more or less compensated by a recovery of quanta in $T_{1}$. A typical case is offered by DAE with small $\phi_{\text {ISC }}$ and $\phi_{t \rightarrow c}$ and high $\phi_{F}$ values (as for $n$-StNs): the quenching can be accompanied here by a gain of reactivity in $T_{1}$, as observed in table 2 for the effect of DCNB on the two DAE bearing a naphthyl group. When, as in the pyrenyl derivative, only $T_{1}$ is reactive (because of a high torsional barrier in $S_{1}$ ) but the triplet yield is very small (because of the competitive fluorescence), the addition of $Q$ can again enhance the $\phi_{t \rightarrow c}$ values through induced ISC.

In polar solvents, such as $\mathrm{MeCN}$, non-emitting CT complexes are formed (Aloisi et al 1991a, 1992; Görner et al 1992). They induce little ISC since the concomitant formation of trans radical ions (generally non-isomerizable) prevails. A parallel increase was generally found both in the absorbance change of the radical ion and the conductivity signal (Aloisi et al 1991a). The photoreaction yield increases slightly in some cases (e.g. with DCNB as acceptor) probably because the radical recombination can lead, at least in part, to formation of the isomerizable triplet state. In any case, the fluorescence quenching by amines was always accompanied by a decrease in the isomerization yield. For example, in the case of 1-StPh/DEA, the $\phi_{\text {CE'im }}$ value was halved (0.07); however, no triplet transient was observed, since $T_{1}$ produced by charge recombination is shorter-lived compared with the radical anion. In some experiments, the radical cations of the olefin were sensitized by irradiation of chloranil, a well-known electron acceptor in the triplet state. Figure 4 refers to the system 1-StPy/Chl in MeCN after irradiation of the acceptor at $308 \mathrm{~nm}$. It shows the spectra of the triplet state of the acceptor $\left(\lambda_{\max }=510 \mathrm{~nm}\right)$ and its radical anion $(450 \mathrm{~nm})$ and of the DAE radical cation $(590 \mathrm{~nm})$ together with the decay kinetics of the first and last transients. The triplet state of $\mathrm{Chl}$ was formed within the laser pulse while the DAE radical reached its maximum $\Delta A$ after $0.6 \mu$ s and decayed with a lifetime of $2 \cdot 5 \mu \mathrm{s}$. 


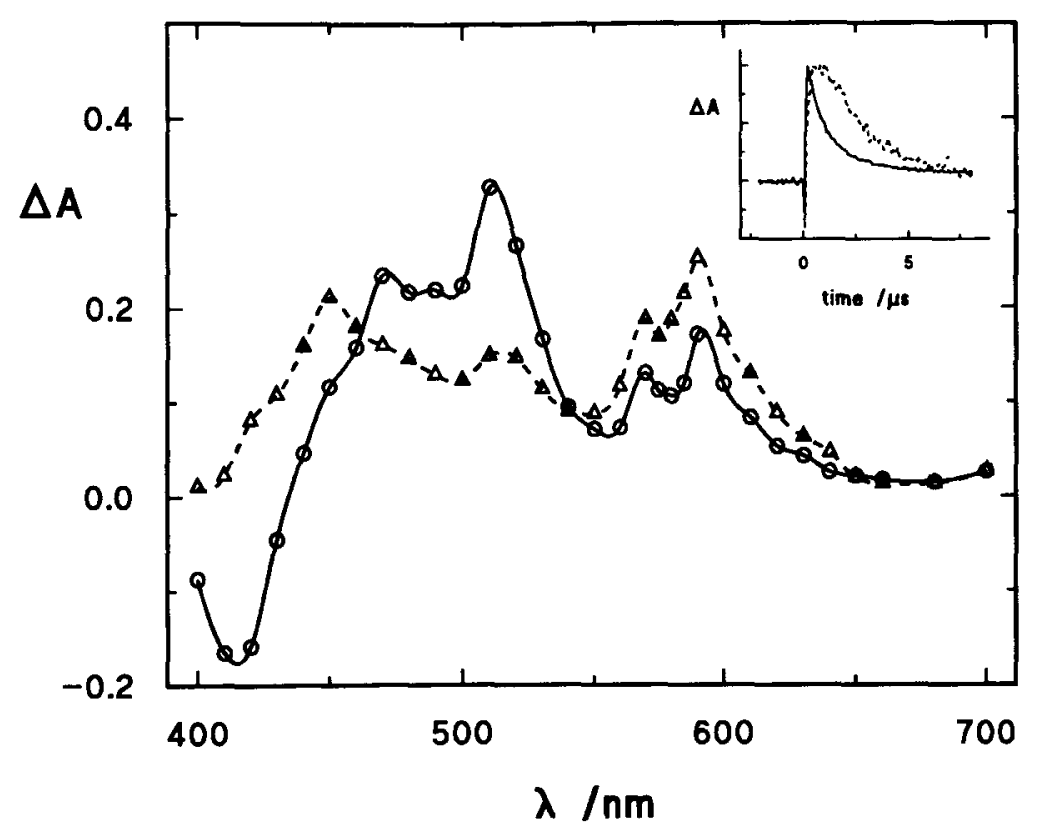

Figure 4. Time-resolved absorption spectra of the trans-1-StPy/Chl system in MeCN recorded $022(--)$ and $1(---)) \mu s$ after the laser pulse $\left(\lambda_{\text {exc }}=308 \mathrm{~nm}\right)$. Inset: decay kinetics at $510(\longrightarrow)$ and $590(---) \mathrm{nm}$.

An interesting case is presented by azastilbenes, and particularly by $1-(n$-pyridyl), 2 -( $n^{\prime}$-pyridyl)ethylenes ( $n, n^{\prime}$-DPE), where the photoreaction is inhibited by the high efficiency of the $S_{1} \rightarrow S_{0}$ internal conversion to the ground state induced by the low-lying $n, \pi^{*}$ states introduced by the heteroatom. Here, the quenching effect is expected to reduce the internal conversion in favour of reactive deactivation pathways through the complex. However, preliminary experiments indicate that the situation is complicated by formation of by-products. In any case, the $S_{1}$ lifetime of DPE is very short, so that high concentrations are needed in order to induce complexation and then isomerization.

An important heavy atom effect was found on using halide anions as quenchers of some DAE and their aza-analogues, the latter in both neutral and protonated forms. Some typical examples showing an important induction of isomerization are reported in table 3. Particularly interesting is the huge increase in reactivity for the two last protonated substrates. Nanosecond laser flash photolysis measurements were carried out to investigate the transients formed by the interaction of iodide anions with some singlet excited $n$-StPhs. No sign of radical anions of the DAE was found but only a substantial production of their $T_{1}$ states. Figure 5 shows the $T_{1} \rightarrow T_{n}$ spectrum of $1-\mathrm{StPh}\left(\lambda_{\max }=460 \mathrm{~nm}\right)$ obtained in the presence of $\mathrm{KI} \sim 0.2 \mathrm{M}$ in $\mathrm{MeCN} / \mathrm{H}_{2} \mathrm{O}(1 / 1, \mathrm{v} / \mathrm{v})$ together with the enhancement of the triplet population by increasing the $\mathrm{KI}$ concentration (inset). The question of the relative importance of the CT and heavy atom effects on the quenching by halide anions has never received a convincing answer (Watkins 1973, 1974). The heavy atom effect probably operates in conjunction with association phenomena, particularly in the case of the charged olefins. In agreement with what was reported for the quaternary salts of DPE (Gutierrez and Whitten 1976), we believe that the actual quenching is controlled by 
Table 3. Limiting trans $\rightarrow$ cis photoisomerization quantum yields of some DAE, their neutral and protonated aza-analogues induced by iodide anions in polar solvents, compared with the intrinsic values in the absence of additives.

\begin{tabular}{|c|c|c|}
\hline Compound & $\phi_{t \rightarrow c}$ & $\phi_{\mathbf{C E}}^{\mathrm{lim}}$ \\
\hline 2-StN ${ }^{a}$ & $0 \cdot 19$ & 0.48 \\
\hline 3-StPh & 0.13 & 0.43 \\
\hline 9-StPh ${ }^{b}$ & 0.29 & 0.40 \\
\hline $3-S_{t} P^{a}$ & 0.44 & 0.52 \\
\hline $2,3^{\prime}-\mathrm{NPE}^{\mathrm{a}}$ & $0 \cdot 14$ & $0 \cdot 38$ \\
\hline $3-\mathrm{StPH}^{+\mathrm{c}}$ & 0.27 & 0.25 \\
\hline $4^{\prime}-\mathrm{OCH}_{3}-3-\mathrm{StPH}^{+c}$ & 0.0006 & 0.36 \\
\hline $3,3^{\prime}-\mathrm{DPEH}_{2}{ }^{++c}$ & 0.045 & $0.50^{\mathrm{d}}$ \\
\hline
\end{tabular}

In water-acetonitrile 60:40, (Mazzucato et al 1982); ${ }^{b}$ in water-acetonitrile 50:50, this work; ${ }^{\text {c }}$ in water-ethanol 90:10 (Bartocci et al 1976/77); ${ }^{\mathrm{d}}$ quencher: bromine anion.

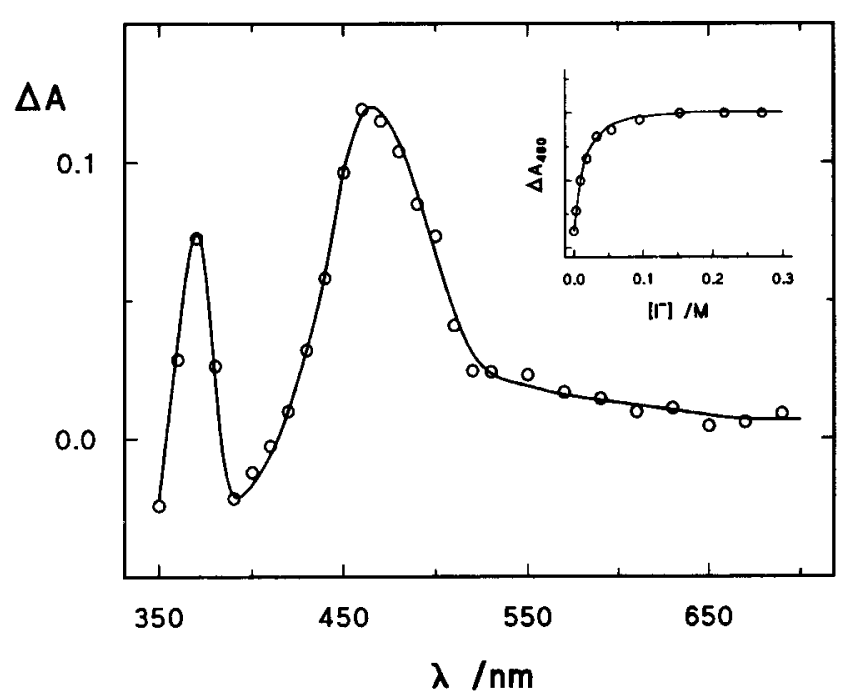

Figure 5. Triplet-triplet absorption spectrum of trans-1-StPh in $\mathrm{MeCN} / \mathrm{H}_{2} \mathrm{O}(1 / 1, \mathrm{v} / \mathrm{v})$ in the presence of $0.5 \mathrm{M} \mathrm{KI}$ recorded $60 \mathrm{~ns}$ after the laser pulse $\left(\lambda_{\text {exc }}=308 \mathrm{~nm}\right)$. Inset: effect of iodide concentration on the absorbance change at $460 \mathrm{~nm}$.

the electron donor-acceptor interaction while the heavy atom makes the triplet formation of the olefin fast enough to dominate all other paths of exciplex decay.

\subsection{Effect of CT interactions on cis $\rightarrow$ trans photoisomerization}

The quenching of the cis-DAE by donor-acceptor interactions has been little investigated thus far since these isomers are generally non-fluorescent due to their fast (small barriers) isomerization to trans or to polycyclic compounds (dihydrophenan- 
threne in the case of $S$ ) and the consequent very short $S_{1}$ lifetime. Since photocyclization is a competitive singlet reaction, CT-induced formation of the triplet state can favour the $c i s \rightarrow$ trans isomerization (particularly for the naphthyl and phenanthryl derivatives which are characterized by substantial cyclization yields). When a maximum is present in ${ }^{1}$ perp*, the ${ }^{1}$ cis* lifetime increases; as mentioned earlier, the twisting becomes more or less activated and leads directly to ${ }^{1}$ trans* by an adiabatic mechanism (Sandros and Becker 1987; Spalletti et al 1991; Mazzucato et al 1993). In such cases, e.g. for the cis isomer of 9-StAn, the CT-induced fluorescence quenching can be measured thus obtaining more quantitative information about the CT interactions. Figure 6 shows the fluorescence spectra of cis-9-StAn quenched by DEA with a Stern-Volmer constant $\left(K_{\mathrm{Q}}=k_{\mathrm{Q}} \tau_{F}\right)$ of 3.2 and $8 \mathrm{M}^{-1}$ in $\mathrm{MCH}(\mathrm{a})$ and $\mathrm{CB}(\mathrm{b})$, respectively. The increase of the fluorescence band of the exciplex is clearly observable in both solvents, particularly in the more polar CB where the emission is weaker but redshifted.

The CT interactions of the cis-DAE in polar solvents open a new way to isomerization since their radical ions can thermally convert to the more stable trans

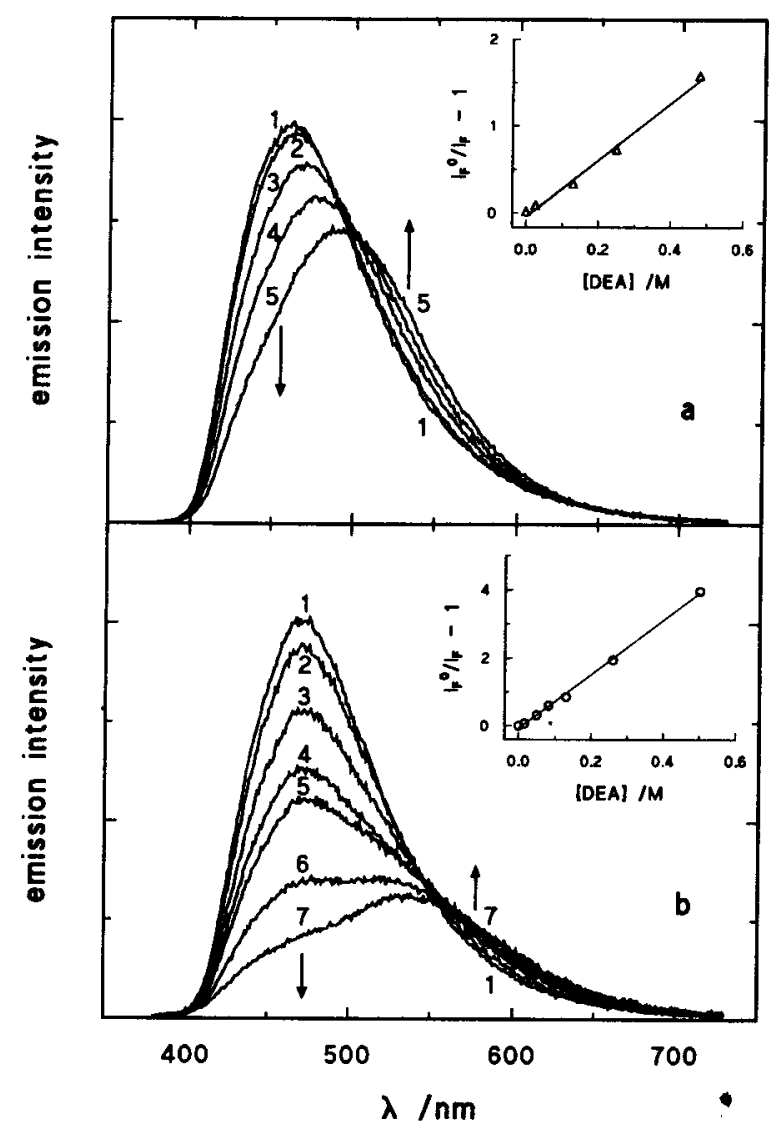

Figure 6. Fluorescence spectra of the cis-9-StAn/DEA system (a) in $\mathrm{MCH}$ without (1) and in the presence of $0.026(2), 0.13(3), 0.25$ (4) and 0.48 (5) MDEA $\left(\lambda_{\text {exc }}=370 \mathrm{~nm}\right)$ [inset: Stern-Volmer plot obtained from the fluorescence intensities recorded at $420 \mathrm{~nm}$ ] and (b) in CB without (1) and in the presence of 0.0164 (2), 0.0492 (3), $0.082(4), 0.13(5), 0.26(6)$ and 0.50 (7) M DEA $\left(\lambda_{\text {exc }}=370 \mathrm{~nm}\right)$ [inset: Stern-Volmer plot obtained from the fluorescence intensities recorded at $470 \mathrm{~nm}$ ]. 


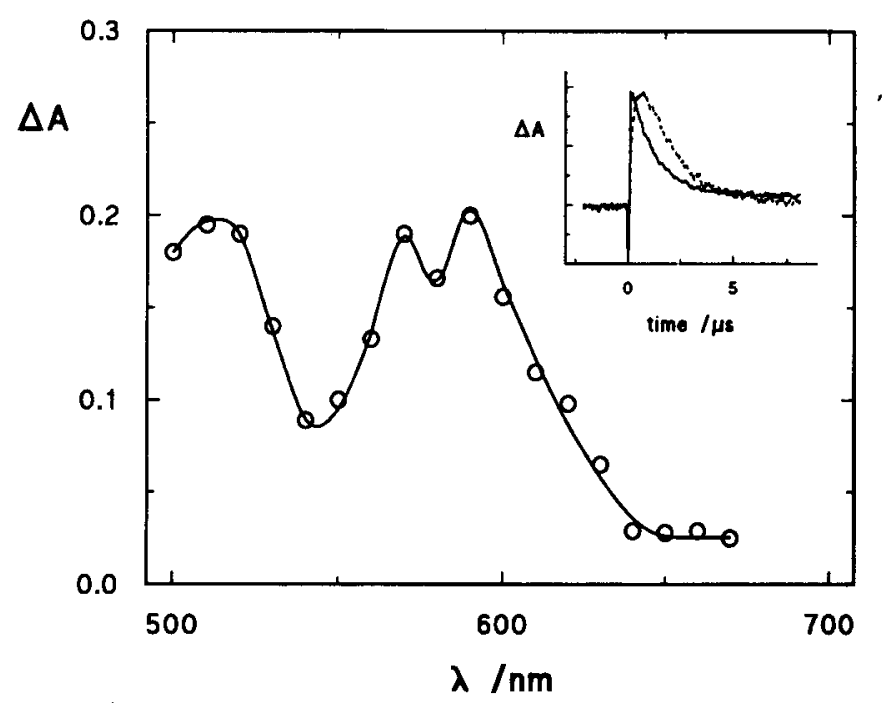

Figure 7. Transient absorption spectrum of the cis-1-StPy/Chl system in $\mathrm{MeCN}$ recorded $0.6 \mu \mathrm{s}$ after the laser pulse $\left(\lambda_{\text {exc }}=308 \mathrm{~nm}\right)$. Inset: decay kinetics at $510(\longrightarrow)$ and $580(-\cdots) \mathrm{nm}$.

radical ions, thus contributing to the overall photoreaction quantum yield. For the cis isomers investigated here (1-StPh, 9-StPh and 1-StPy), laser irradiation of cis-DAE/Chl produced the radical cation in the trans configuration (as indicated by comparison with the transients obtained after direct sensitization of the trans isomers and by its mono-exponential decay) due to the fast cis $^{+} \rightarrow$ trans $^{+}$twisting. Such behaviour is different from that found for cis-stilbene in the presence of Chl and reported with different acceptors by other authors (Lewis et al 1985; Tokumaru et al 1991; Kuriyama et al 1992), which showed biexponential decay assigned to the cis $^{*}$ (shorter-lived) and trans ${ }^{+}$(longer-lived) contributions. This difference can be due to a smaller barrier to twisting for the radical cations of the present compounds. A study of the temperature effect is in progress to confirm such hypothesis. Figure 7 shows an example for the system cis-1-StPy/Chl in $\mathrm{MeCN}$. The transient spectrum at room temperature was assigned to the radical cation in the trans configuration (see figure 4) formed after ${ }^{3} \mathrm{Chl}^{*}$ sensitization of the cis cation. The trans ${ }^{+}$absorption was found to increase by addition of $\mathrm{LiClO}_{4}$ and by increasing the cis concentration (chain mechanism) as reported for cis-stilbene (Lewis et al 1985). Such effects indicate that the alkali metal cation protects the olefin radical cation from recombination with the acceptor radical anion (back transfer) (Lewis et al 1985) or with the superoxide anion (formed by $\mathrm{Chl}^{-}+\mathrm{O}_{2}$ ) (Kuriyama et al 1992; Tokumaru et al 1991).

\section{Acknowledgement}

Financial support by the Italian Consiglio Nazionale delle Ricerche (Progetto Finalizzato Chimica Fine e Secondaria II) and Ministero per l' Università e la Ricerca Scientifica e Tecnologica (Rome) is gratefully acknowledged. 


\section{References}

Aloisi G G, Bartocci G, Favaro G and Mazzucato U 1980 J. Phys. Chem. 842020

Aloisi G G and Elisei F 1990 J. Phys. Chem. 945813

Aloisi G G, Elisei F and Görner H 1991a J. Phys. Chem. 954225

Aloisi G G, Elisei F and Latterini L 1992 J. Chem. Soc., Faraday Trans. 882139

Aloisi G G, Elisei F, Mazzucato U and Prats M 1991b J. Photochem. Photobiol. A62 217

Aloisi G G, Masetti F, Elisei F and Mazzucato U 1988 J. Phys. Chem. 923394

Aloisi G G, Mazzucato U, Birks J B and Minuti L 1977 J. Am. Chem. Soc. 996340

Arai T, Karatsu T, Misawa H, Kuriyama Y, Okamoto H, Hiresaki T, Furuuchi H, Zeng H, Sakuragi H and Tokumaru K 1988 Pure Appl. Chem. 60989

Bartocci G, Masetti F, Mazzucato U and Marconi G 1984 J. Chem. Soc., Faraday Trans. 2, 801093

Bartocci G, Masetti F, Mazzucato U, Spalletti A, Baraldi I and Momicchioli F 1987 J. Phys. Chem. 914733

Bartocci G, Mazzucato U and Bortolus P 1976/77 J. Photochem. 6309

Bartocci G, Mazzucato U, Spalletti A, Orlandi G and Poggi G 1992 J. Chem. Soc., Faraday Trans. 883139

Elisei F, Aloisi G G and Masetti F 1992 J. Chem. Soc., Faraday Trans. 882155

Elisei F, Aloisi G G and Mazzucato U 1990 J. Phys. Chem. 945818

Elisei F, Mazzucato U and Görner H 1989 J. Chem. Soc., Faraday Trans. 1851469

Görner H, Elisei F and Aloisi G G 1992 J. Chem. Soc., Faraday Trans. 8829

Görner H and Schulte-Frohlinde D 1979 J. Phys. Chem. 833107

Gutierrez A R and Whitten D G 1976 J. Am. Chem. Soc. 986233

Kuriyama Y, Arai T, Sakuragi H and Tokumaru K 1992 Chem. Lett. 879

Lewis F D 1979 Acc. Chem. Res. 12152

Lewis F D, Petisce J R, Oxman J D and Nepras M J 1985 J. Am. Chem. Soc. 107203

Lewis F D, Reddy G D and Bassani D 1992 J. Photochem. Photobiol. A65 205 and references therein

Lewis F D and Simpson J T 1979 J. Phys. Chem. 832015

Mazzucato U 1982 Pure Appl. Chem. 541705

Mazzucato U $1987 \mathrm{Gazz}$. Chim. Ital. 117661

Mazzucato U, Aloisi G G and Masetti F 1982 J. Photochem. 18211

Mazzucato U and Momicchioli F 1991 Chem. Rev. 911679

Mazzucato U, Spalletti A, Bartocci G and Galiazzo G 1993 Coord. Chem. Rev. 125251

Saltiel J and Charlton J L 1980 Rearrangements in ground and excited states (ed.) P de Mayo (New York: Academic Press) vol. 3, p. 25

Saltiel J and Sun Y -P 1990 Photochromism: Molecules and systems (eds) H Dürr and H Bouas-Laurent (Amsterdam: Elsevier) p. 64

Sandros K and Becker H -D 1987 J. Photochem. 39301

Spalletti A, Bartocci G, Mazzucato U and Galiazzo G 1991 Chem. Phys. Lett. 186297

Tokumaru K and Arai T 1992 J. Photochem. Photobiol. A65 1

Tokumaru K, Kuriyama Y, Arai T, Lednev I K, Akaba R and Sakuragi H 1991 Photochemical processes in organized molecular systems (ed.) K Onda (Amsterdam: Elsevier) p. 199

Watkins A R 1973 J. Phys. Chem. 771207

Watkins A R 1974 J. Phys. Chem. 78 1885, 2555 